www.jmscr.igmpublication.org

Impact Factor 5.84

Index Copernicus Value: 71.58

ISSN (e)-2347-176x ISSN (p) 2455-0450

crossref DOI: _https://dx.doi.org/10.18535/jmscr/v5i9.121

Journal Of Medical Science And Clinical Research

\title{
Review of Shunthi (Zingiber officinale Rosc.) in Ayurvedic Literature
}

\author{
Authors \\ Dr Anant Saznam ${ }^{1}$, Dr Satyendra Kumar Singh ${ }^{2}$ \\ ${ }^{1}$ Lecturer, Dravyguna Deptt. SRTAC and H Karjara, Gaya, Bihar \\ ${ }^{2}$ Ayurvedic Medical officer, Ayush Bihar
}

\begin{abstract}
Shunthi (Zingiber officinale Rosc.) belongs to Zingiberaceae family. It is a perennial erect herb with a creeping tuberous rhizome. Root stock horizontal, tuberous, aromatic, stout, rhizome with erect leafy stems 0.6-1.4 meter high. Stem elongated, leafy, $15-150 \mathrm{~cm}$. tall. Leaves narrow, linear, sessile and sub sessile on the sheaths, with an alternative base, acuminate, glabrous, $10-50 \mathrm{~cm}$. long lower part surrounding the stem, 5-10 inch long, smooth, ligule glabrous and sheaths glabrous. Flowers- shoot up to $12 \mathrm{~cm}$ long, clothed with sheaths; bracts 2-5 cm $\times 2 \mathrm{~cm}$, light green; corolla tube light yellow, lip orbicular, dull purple with creamy blotches. Flowering and fruiting during July-September. It is cultivated almost throughout India. Shunthi, mahaushdh, vishw, nagar, vishwbheshaj, vishvaushadh, katugranthi, katubhadr, katushn, sauparn, shringver, kafari, aardrak, shoshan, nagar are its various synonyms found in ayurvedic texts. Rasa -Katu, Guna - snigdha, virya - ushna, vipaka - Madhura are properties of this drug. Drug is shothahara and Vatakaphahara and used as deepana and bhedana. It is used in various disease like shula, amavata, aadhyamana, atisara, shlipada, kasa, shwasa, hridroga, shopha, hikka, vibandha, rakttapita, pandu, vrana, jvara, kustha, agnimandya etc.
\end{abstract}

Keywords: Shunthi, Zingiberacea, Vata-Kafahara.

\section{INTRODUCTION}

Ayurvedic medicine is one of the world oldest medicine systems. It originated in India more than 5000 years ago remains one of the traditional health care system .The term Ayurveda combines the Sanskrit words Ayur (life), Veda (science or knowledge). The aim of Ayurveda is to prevent illness, heal the sick and preserve life. This can be summed up of follows,

To protect health and prolong life 'Swasthya sswasthya rakshanam'.

To eliminate diseases and dysfunction of the body

'Aturasya vicar prashamanamcha'.

Dravyaguna is an essential part, of Ayurveda. Dravyaguna vigyana depends upon the following basic principles i.e. Rasa (taste including chemical composition), guna (physical properties), virya (dynamic property), vipak (metabolic property), prabhava (specific properties), karma (action).

Name, synonyms, morphology, rasa, guna, virya, vipaka, prabhava, and karma of drugs are mentioned in drayaguna vigyana. shunthi is mentioned in various ayurvedic literature. A review regarding the literature of present study drug Shunthi is presented as references available from various Ayurvedic literature.

\section{VEDIC GRANTHAS}

There are very few descriptions seen about Shunthi as Visvabhesaja, Mahausadh etc. In Guhayasutra the word Shunthi is being used as a 
Trinaveshesha i.e., described as a representative drug for kusha. This word Shunthi is used for 'Alpakaya' or 'Hrasva'.

\section{SAMHITA GRANTHAS}

In samhita granthas Shunthi has been mentioned at numerous places for various diseases i.e. Jvara, Kasa, Gulma, Amavata etc. in different formulations.

\section{CHARAKA SAMHITA: (1000B.C- $4^{\text {th }}$ Cent.A.D)}

In Charaka samhita name of Shunthi has appeared first time in sutrasthana $4^{\text {th }}$ chapter; here this is used in Mandagni as an ingredient of Deepaniya mahakasaya. Shunthi is placed in Deepaniya, Mahakasaya, Trptighna, Mahakasaya, Arsoghna, Mahakasaya, Sitaprasamana Mahakasaya and sula Prasama Mahakasaya. The name of Preparations, indications, and references of Shunthi are enlisted below.

Table no.1

\begin{tabular}{|c|c|c|c|}
\hline S.N. & Preparation/group & Indiations $\backslash$ Actions & References \\
\hline 1 & Deepaniya Mahakasaya & Deepana, Mandagni & Su. $4 / 6$ \\
\hline 2 & Trptighana Mahakasaya & Aruchi & Su.4/11 \\
\hline 3 & Arsoghana Mahakasaya & Arsa & $\mathrm{Su} 4 / 12$ \\
\hline 4 & Sitaprasamana Mahakasaya & Jvara & $\mathrm{Su} 2 / 42$ \\
\hline 5 & Sulaprasamana Mahakasaya & Shula & $\mathrm{Su} 4 / 45$ \\
\hline 6 & Siddha taila & Snehana & Su13/86 \\
\hline 7 & Kwatha & Krmiapakarsana & $\mathrm{Vi} 7 / 17$ \\
\hline 8 & Yavagu & Krmiapakarsana & Vi7/19 \\
\hline 9 & Katuskanda & Kaphaja Roga & Vi8/142 \\
\hline 10 & Sirovirecana Dravyakalpsamgraha & Sirovirecana & Vi8/151 \\
\hline 11 & Agurvadya taila & Jvara & Chi3/267 \\
\hline 12 & Pippalyadyaghrta & Vatikgulma & Chi5/74 \\
\hline 13 & Yavagu & Atisara & Chi.8/125 \\
\hline 14 & Pathyadi Churna & Yakshma & Chi8/126 \\
\hline 15 & Saindhavadi Churna & Kshata Kshina Chikitsa & Chi11/85 \\
\hline 16 & Sadava & Yakshma Roga & Chi11/88 \\
\hline 17 & Shunthi Kalpa & Kshata Kshina Chikitsa & Chi11/92 \\
\hline 18 & Kwatha & Svayathu Chikitsa & Chi12/24 \\
\hline 19 & Kwatha & Kaphaja Shotha & Chi12/70 \\
\hline 20 & Vidangadi Kshara & Udararoga Chikitsa & Chi13/80 \\
\hline 21 & Shunthi Churna & Kaphodara & Chi13/104 \\
\hline 22 & Nagaradi Churna & Udararoga & Chi13/115 \\
\hline 23 & Shunthi kshira & Udararoga & Chi13/153 \\
\hline 24 & Kshara vatika & Udararoga & Chi13/163 \\
\hline 25 & Peya & Shushkarsa & Chi14/89 \\
\hline 26 & Pippalyadi Ghrita & Arsa & Chi14/104 \\
\hline 27 & Pippalimuladi Ghrita & Arsa & Chi14/105 \\
\hline 28 & Dadimadi Ghrita & Panduroga & Chi16/44 \\
\hline 29 & Duralabhadileha & Kasa Chikitsa & Chi18/50 \\
\hline 30 & Saindhavadi Yoga & Kasa Chikitsa & Chi18/63 \\
\hline 31 & Kwatha & Kasa Chikitsa & Chi18/112 \\
\hline 32 & Pathyadi kalka & Kasa Chikitsa & Chi18/114 \\
\hline 33 & Nagaradi Kalka & Kasa Chikitsa & Chi18/115 \\
\hline 34 & Pacaka Jala & Atisara & Chi19/22 \\
\hline 35 & Dravyadi Ghrita & Atisara & Chi19/80 \\
\hline 36 & Drakshadi Sitakasaya & Visarpa Chikitsa & Chi21/58 \\
\hline 37 & Churna & Bicchhu Chikitsa & Chi23/208 \\
\hline 38 & Bijapurakadi Madya & Madayata Chikitsa & Chi24/121 \\
\hline 39 & Hingwadi Churna & $\begin{array}{l}\text { Pliha,Udararoga, } \\
\text { Ajirna,Visucika }\end{array}$ & Chi26/22 \\
\hline 40 & Kaserukadi Ghrita & Pittajahrdayaroga & Chi26/94 \\
\hline
\end{tabular}




\begin{tabular}{|l|l|c|c|}
41 & Katphaladi Kashaya & Kaphajahrdayaroga & Chi26/97 \\
\hline 42 & Krsnadi Churna & Kaphajahrdayaroga & Chi26/97 \\
\hline 43 & Kshira paka & Pittajapinasa & Chi26/144 \\
\hline 44 & Hingvadi taila & Trimarmiya Chikitsa & Chi26/222 \\
\hline 45 & Devadarvadi Taila & Trimarmiya Chikitsa & Chi26/223 \\
\hline 46 & Gandha Taila & Trimarmiya Chikitsa & Chi26/224 \\
\hline 47 & Kshara Taila & Trimarmiya Chikitsa & Chi26/226 \\
48 & Saindhavadi Taila & Urustambha Chikitsa & Chi27/45 \\
\hline 49 & Svadanstra Taila & Vatavyadhi Chikitsa & Chi28/147 \\
\hline 50 & Mulaka Taila & Vatavyadhi Chikitsa & Chi28/168 \\
\hline 51 & Sukumaraka Taila & Vatavyadhi Chikitsa & Chi29/99 \\
\hline 52 & Pusyanuga Churna & Yonivyapat Chikitsa & Chi30/92 \\
\hline 53 & Pancakoladilepa & Yonivyapat Chikitsa & Chi30/264 \\
\hline
\end{tabular}

SUSURATA SAMHITA (1000B.C-5 ${ }^{\text {th }}$ cent. He placed Shunthi in Pippalyadi Gana and A.D) trikatu. $^{2}$

First of all Shusurta has mentioned Shunthi in

Shutrasthana Chapter $38^{\text {th }}$ in Pratisaraniya Kshara.

Table no.2

\begin{tabular}{|c|c|c|c|}
\hline $\begin{array}{l}\text { S.N. } \\
1 \\
2\end{array}$ & $\begin{array}{l}\text { Preparations/group of drugs } \\
\text { Pippalayadi Gana } \\
\text { Trikatu }\end{array}$ & $\begin{array}{c}\text { Indications/Actions } \\
\text { Pratisaya, Aruchi } \\
\text { Prameha, Kustha, Tvakaroga }\end{array}$ & $\begin{array}{c}\text { References } \\
\text { Su38/22 } \\
\text { Su38/58 }\end{array}$ \\
\hline 3 & Virechana dravya & Slesma Vikara & $\mathrm{Su} 44 / 20$ \\
\hline 4 & Churna & Virechana & Su44/65 \\
\hline 5 & Besavara & Vata Vikara & Su46/369 \\
\hline 6 & Churna & Ajirna & Su.46/519 \\
\hline 7 & Siddha Kshira & Vata Vyadhi & Sa.10/18 \\
\hline 8 & Siddha Kshira & Vata vyadhi & Sa.10/68 \\
\hline 9 & Kwatha & Vataja vatarakta & Chi5/7 \\
\hline 10 & Kalka & Kaphaja Vatarakta & Chi5/10 \\
\hline 11 & Swarasa & Vatavyadhi, karnashula & Chi5/24 \\
\hline 12 & Takra & Arsa Chikitsa & Chi6/13 \\
\hline 13 & Siddha Kshira & Kaphaja Arsa & Chi6/16 \\
\hline 14 & Dhanavanatra Ghrita & Prameha Pidika & Chi12/5 \\
\hline 15 & Churna & Virechana & Chi14/10 \\
\hline 16 & Satpala Ghrita & Udararoga & Chi14/14 \\
\hline 17 & Churna & Mudhagarbha & Chi15/20 \\
\hline 18 & Churna & Galagandaroga & Chi18/51 \\
\hline 19 & Churna & Vidradhi Chikitsa & Chi16/33 \\
\hline 20 & Kalka & Kaphajaslipada Chikitsa & Chi19/57 \\
\hline 21 & Churna & Shopha Chikitsa & Chi23/12 \\
\hline 22 & Churna & Chhuchhndra visa & Ka.7/17 \\
\hline 23 & Kalka & Svedanartha & Ка. $8 / 45$ \\
\hline 24 & Anjana & Shushkakshipaka & U.9/20 \\
\hline 25 & Lepa & Slesmabhisyanda & U.11/6 \\
\hline 26 & Anjana & Netrakandu Shopha & U.11/17 \\
\hline 27 & Anjana & Netrapaka & U.12/44 \\
\hline 28 & Lepa & Netravedna, Lalima & U.17/90 \\
\hline 29 & Swarasa & Karnashula & U.21/18 \\
\hline 30 & Swarasa & Karnashula & U.21/25 \\
\hline 31 & Churna & Atisara & U.40/36 \\
\hline 32 & Churna & Amatisara & U.40/42 \\
\hline 33 & Churna & Amatisara & U.40/49 \\
\hline
\end{tabular}




\begin{tabular}{|l|l|c|c|}
34 & Churna & Pittatisara & U.40/65 \\
\hline 35 & Churna & Pakvatisara & U.40/71 \\
\hline 36 & Churna & Atisara & U.40/132 \\
\hline 37 & Churna & Pravahika & U.40/154 \\
\hline 38 & Churna & Kaphajasula & U.42/110 \\
\hline 39 & Churna & Vatajahridaroga & U.43/12 \\
40 & Kamala Chikitsa & U.44/33 \\
41 & Churna & Panajirna & U47/38 \\
\hline 42 & Churna & Trisnasamaka & U.47/80 \\
\hline 43 & Kshirapaka & Hikkahara & U50/23 \\
\hline 44 & Srngayadi Ghrita & Swasa, Kasa, & U.51/21 \\
\hline 45 & Leha & Swasa, Kasa & U.51/34 \\
\hline 46 & Phalatrikadi Curna & Kasahara & U.52/15 \\
\hline 47 & Pathyadi Curna & Kasahara & U.52/16 \\
\hline 48 & Devadarvadi Kwatha & Udavarta & U.55/45 \\
\hline 49 & Sirsadi Varti & Grabhada & U.60/43 \\
\hline
\end{tabular}

ASTANGA HRDAYA (700A.D)

Table no.3

\begin{tabular}{|c|c|c|c|}
\hline S.N. & Preparations/group of drug & Indications/Actions & References \\
\hline 1 & Swarasa & Pratishyaya & Su. $3 / 23$ \\
\hline 2 & Katu rasa & Vrishya & Su.10/35 \\
\hline 3 & Siddha Jala & Pacana, Jvarahara & chi.1/15 \\
\hline 4 & Peya & Hitakara in Jvara & Chi1/26 \\
\hline 5 & Peya & Atisara & Chi $1 / 27$ \\
\hline 6 & Sita Kashaya & Jvara, Pinasa, Kasa & Chi.1/45 \\
\hline 7 & Kwatha & Kapha jvara & Chi1/54 \\
\hline 8 & Kwatha & Pitta kapha jvara & Chi1/61 \\
\hline 9 & Kwatha & Pitta kapha jvara & Chil/62 \\
\hline 10 & Mansarasa & Jvara & Chi1/77 \\
\hline 11 & Siddha dugdha & Trisna, Daha, Jvara & Chi1/109 \\
\hline 12 & Siddha dugdha & Jvara, Kasa & Chi.1/114 \\
\hline 13 & Peya & Raktapitta & Chi. 2/16 \\
\hline 14 & Siddha Kshira & Gudmargagami Raktapitta & Chi.2/40 \\
\hline 15 & Churna & Vataj Kasa & Chi.3/13 \\
\hline 16 & Churna & Kasa & Chi.3/15 \\
\hline 17 & Leha & Kaphaja Kasa & Chi.3/46 \\
\hline 18 & Amritaprasa Ghrita & Kasa & Chi.3/94 \\
\hline 19 & Kusmanda rasayana & Kasa Chikitsa & Chi.3/115 \\
\hline 20 & Sadava Churna & Kasa & Chi.3/145 \\
\hline 21 & Churna & Kasa & Chi3/174 \\
\hline 22 & Churna & Swasa, Hikka Cikitsa & Chi4/32 \\
\hline 23 & Churna & Rajayakshma & Chi.5/45 \\
\hline 24 & Kalka & Vataja Chhardi & Chi.6/9 \\
\hline 25 & Kwatha & Parsvashula, Hridroga, Swasroga & Chi.6/28 \\
\hline 26 & Dadimadi churna & Apatantraka, Hirdoga, Swasaghna & Chi6/30 \\
\hline 27 & Siddha Jala & Trinsa & Chi6/36 \\
\hline 28 & Siddha Ghrita & Paittika Hridoga & Chi6/48 \\
\hline 29 & Churna & Hridroga & Chi6/52 \\
\hline 30 & Kwatha & Vataja Madatya & Chi7/17 \\
\hline 31 & Kwatha & Madatya & Chi7/26 \\
\hline 32 & Sita kashaya & Kaphaja Madatya & Chi7/33 \\
\hline 33 & Mansarasa & Kaphaja Madatya & Ci7/38 \\
\hline 34 & Kasisadi Taila & Arsa & Chi8/15 \\
\hline 35 & Peya & Arsa & Chi8/86 \\
\hline 36 & Kwatha & Raktarsa & Chi8/102 \\
\hline 37 & Yavanyadi Churna & Raktarsa & Chi8/115 \\
\hline
\end{tabular}




\begin{tabular}{|c|c|c|c|}
\hline 38 & Kwatha & Atisara & Chi $9 / 6$ \\
\hline 39 & Siddha Ghrita & Pakwatisara & Chi9/18 \\
\hline 40 & Kwatha & Pittatisara & Chi9/58 \\
\hline 41 & Pathadi Kwatha/churna & Slesmatisara & Chi9/105 \\
\hline 42 & Talisadi Vati & Grahani & Chi10/16 \\
\hline 43 & Shunthyadi yoga & Gulma & Chi14/41 \\
\hline 44 & Chitrakadi kwatha & Shula, Anaha, vibandha & Chi14/48 \\
\hline 45 & Danti-Haritaki Paka & Gulma & Chi14/94 \\
\hline 46 & Hingavadi Kashara & Udararoga & Chi15/71 \\
\hline 47 & Ksharagutika & Ajirna, Shotha, Udarroga & Chi15/104 \\
\hline 48 & Takra & Jalodra & Chi15/128 \\
\hline 49 & Drakshadi Awaleha & Pandu and kamla & Chi16/30 \\
\hline 50 & Churna & Svayathu & Chi17/2 \\
\hline 51 & Lepa & Ekanga Shopha & Chi1729 \\
\hline 52 & Churna & Vata-Kaphaja Hridaya -Shula & Chi21/37 \\
\hline 53 & Churna & Pravahika & Ka.3/15 \\
\hline 54 & Kwatha & Kshiralasaka & $\mathrm{U} .2 / 25$ \\
\hline 55 & Kalka & Talukantaka & U.2/66 \\
\hline 56 & Churna & Pothaki & U.9/21 \\
\hline 57 & Nasaya & Kaphaja Timara & U.13/68 \\
\hline 58 & Ghrita Paka & Putaka & U.20/18 \\
\hline 59 & Churna & Upkusha & U.22/31 \\
\hline 60 & Siddha kshira & Vataja Slipada & U.30/10 \\
\hline 61 & Pusyanuga Churna & Yoni roga & U.30/47 \\
\hline 62 & Shuthyadi Rasayana & Rasayana & U.39/104 \\
\hline
\end{tabular}

BHELA SAMHITA: (1000B.C)

\section{Table no.4}

\begin{tabular}{|l|l|c|c|}
\hline S.N. & Preparations/group of drugs & Indications/Actions & References \\
\hline 1 & Nagaradi Ghrita & Vishama Jvara & Chi2/20 \\
\hline 2 & Tryusanadi Ghrita & Grahani Arsa & Chi.2/23 \\
\hline 3 & Dasanga Ghrita & Kasa, Swasa & Chi.5/17 \\
4 & Dadhika Ghrita & Rakta Gulma & Chi.5/25 \\
\hline 5 & Satpala Ghrita & Grahni & Chi5/32 \\
\hline 6 & Churna & Prameha & Chi7/11 \\
\hline 7 & Dasamulad Taila & Slipada & Chi.14/16 \\
\hline 8 & Changeri Ghrita & Raktarsa & Chi16/44 \\
\hline 9 & Dantyadyayasa & Svayathu & Chi $17 / 21$ \\
\hline 10 & Churna & Atisara, Kasa & Chi17/35 \\
\hline
\end{tabular}

\section{KASYAPA SAMHITA;(600 A.D)}

\section{Table no.5}

\begin{tabular}{|l|l|c|c|}
\hline S.N. & Preparation/group of drugs & Indication/action & References \\
\hline 1 & Ghrita & Lehan & Lehadhyaya \\
\hline 2 & Churna & Dugdha- Shodhana & Ksirotpatthdhyaya/19 \\
\hline 3 & Swarasa & Dugdha- Shodhana & Ksirotpatthdhyaya/19 \\
4 & Churna & Dipana & Garbhini Chikitsa \\
\hline 5 & Ghrita & Sita Putana & Balagraha Chikitsa \\
\hline 6 & Narachaka & Gulma,Arsa & Udavartta Chikitsa \\
\hline 7 & Dasanga Ghrita & Vata gulma & Gulma Chikitsa/23 \\
\hline 8 & Shatpala Ghrita & Sarwa gulma & Gulma Chikitsa/25 \\
\hline 9 & Siddha Kshira & Mutraja mutrakrichhra & Mutrichra chikitsa \\
\hline
\end{tabular}




\section{CHIKITSA GRANTHAS}

\section{VRINDA MADHAV}

\section{Table no.6}

\begin{tabular}{|l|l|c|c|}
\hline S.N. & Preparations/groups of drugs & Indications/Actions & References \\
\hline 1 & Rasnadi Kashaya & Amapachana & Chi.25/2 \\
\hline 2 & Rasnad pancaka Kwatha & Sarvangavata, Amavata & Chi.25/10 \\
\hline 3 & $\begin{array}{l}\text { Rasnadisaptaka Kwatha With } \\
\text { Nagarachurna }\end{array}$ & $\begin{array}{c}\text { Janghorutrikpristha- Parsvashula } \\
\text { Amavata }\end{array}$ & Chi25/11 \\
\hline 4 & Shunthi- Gokshura Kwatha & Samavata, katishula Pachana & Chi25/18 \\
\hline 5 & Vaisvanara Churna & Amavata,Gulma, Hridroga & Chi25/26 \\
\hline 6 & Yogaraja & Amavata, Adhyavata, Krimi & Chi25/36 \\
\hline 7 & Ajmodadi Vataka & Visvachi, Pratituni, Tuni & Chi25/63 \\
\hline 8 & S unthi Ghrita & Vataslesma Prasamana, Agnisandipan & Chi25/69 \\
\hline
\end{tabular}

\section{CHAKRADATTA: $\left(11^{\text {th }}\right.$ Century A.D)}

\section{Table no.7}

\begin{tabular}{|l|l|c|c|}
\hline S.N & Preparations/groups of drugs & Indications/Actions & References \\
\hline 1 & Sadangapaniya & Jvara & JvaraChikitsa-2/19 \\
\hline 2 & Visvadi Kashaya & Pitta jvara & Jvara Chikitsa-2/88 \\
\hline 3 & Sunthyadi Kwatha & Amadosa Grahani & Grahni-4/6 \\
\hline 4 & Kalyanaka leha & Jadya, Gadagada, Muktva & Vatavyadhi-22/20 \\
\hline 5 & Trayodasanga Guggulu & $\begin{array}{c}\text { Katigraha, Gridhrasi, Kushtha } \\
\text { Sampurna Vataroga }\end{array}$ & Vatavyadhi-22/69 \\
Vatavyadhi-22/152 \\
\hline 7 & Saindhavadya Taila & Amayuktavatarakta & Vatarakta-23/3 \\
\hline 8 & Amritadi Kwatha & Muktrakrichhra, Udavarta, & Vataraktadhikara23/26 \\
& Visamajvara & \\
\hline 9 & Amrita Guggulu & Vatarakta, Kushtha, Dustavrana & Vataraktadhikara23/53 \\
\hline 10 & Punarnava Guggulu & Vatarakta, Amavata & Vataraktadhikar-23/60 \\
\hline 11 & Satyadi kwatha & Amavata & Amavatadhikara-25/3 \\
12 & Punarnava Kwatha & Amavata & Amavatadhikara-25/3 \\
\hline 13 & Rasnadasamula kwatha & Amavata & Amavatadhikara-25/3 \\
\hline 14 & Rasanasaptaka Kwatha & Shula in Jaghanapristha, Parava & Amavatadhikara-25/3 \\
& & Trika & \\
\hline 15 & Vaisvanara Churna & Amavata & Amavatadhikara-25/3 \\
\hline 16 & Hingvadi Churna & Amavata & Amavatadhikara-25/3 \\
17 & Yogaraja Guggulu & Amavata & Amavatadhikara-25/3 \\
\hline 18 & Brihatsaindhavadya & Amavata & Amavatadhikara-25/3 \\
\hline 19 & Guduchi Ghrita & Amavata, Adhyavata & Amavatadhikara-25/3 \\
\hline 21 & Hingavadi Vati & Shula & Suladhikara 26/18 \\
\hline 21 & Shunthi kwatha & Hridaya shula, Parsashula & Suladhikara26/87 \\
\hline 22 & Kankayana Gutika & Gulma, Krimi, Hridroga & Gulmadhikara-27/52 \\
\hline 23 & Nagara kwatha & Vataja Hridroga & Hrdrogadhikara-31/4 \\
\hline
\end{tabular}

VANGASENA:

\section{Table no.8}

\begin{tabular}{|l|l|c|c|}
\hline S.N. & Preparations/groups of drugs & Indications/Actions & References \\
\hline 1 & Kwatha & Jvarahara & JvaraChi-4/208 \\
\hline 2 & Kwatha & Vatikajvara & JvaraChi-4/224 \\
\hline 3 & Kwatha & Chhardi, PittaJvara & JvaraChi-4/247 \\
\hline 4 & Kwatha & Swasa, Kasa, Vidbheda & JvaraChi-4/254 \\
\hline 5 & Kwatha & Vatapitta Jvara & JvaraChi-4/291 \\
\hline 6 & Panchatikta Kwatha & All types of jvara & JvaraChi-4/318 \\
\hline 7 & Churna & Sannipataja Jvara & JvaraChi-4/431 \\
\hline 8 & Mustadi Ghana & Krimi, Swayathu, Pandu & JvaraChi-4/476 \\
9 & Nagaradi Vatika & AtisaraChi-5/150 \\
10 & Shunthi Putapaka & AtisaraChi-5/182 \\
\hline
\end{tabular}




\begin{tabular}{|l|l|c|c|}
\hline 11 & Mahabilva Taila & Atisara & AtisaraChi-5/182 \\
\hline 12 & Kwatha & JirnaAma, Grahani & Grahani Chi-6/25 \\
\hline 13 & Kwatha & Dipana, Pachana & Grahani Chi-6/28 \\
\hline 14 & Shunthi Ghrita & Grahani, Pandu, Pliha & Grahan chi-6/58 \\
\hline 15 & Nagaradha Churna & Pittaja Grahani & Grahani chi-6/70 \\
\hline 16 & Kalyana Guda & Grahani, Swasa, Kasa & Grahani Chi-6/200 \\
17 & Udakashatpala Ghrita & Arsa Chi-7/78 \\
18 & Chandanadi Churna & Rakta pitta, Arsa, Jvara & Raktapitta Chi-11/94 \\
\hline 20 & Talisadya Churna & Rajayakshma & Rajyakshma Chi14/75 \\
\hline 21 & Churna & Amavata & AmavataChi-31/26 \\
\hline 22 & Kwatha & Amavata & Amavata Chi-31/27 \\
\hline 23 & Amritadi Churna & Amavata & Amavata Chi-31/48 \\
\hline 24 & Maharasanadi Kwatha & Amavata & Amavata Chi-31/48 \\
\hline 25 & Abhayadi Churna & Amavata & Amavata Chi-31/48 \\
\hline 26 & Shunthi Ghrita & Amavata Chi-31/48 \\
\hline
\end{tabular}

\section{GADANIGRAHA: $\left(12^{\text {th }}\right.$ Century $)$}

Table no.9

\begin{tabular}{|l|l|c|c|}
\hline S.N & Group of drugs/Preparations & Indications/Action & References \\
\hline 1 & Shunthyadi Pachana Kashaya & Amajvara & Jvaradhikar-1/191 \\
2 & Guduchyadi Pachana Kashaya & Jvarahara & Jvaradhikar-1/193 \\
\hline 3 & Shunthyadi Kwatha & Vatajvara & Jvaradhikar-1/219 \\
\hline 4 & Dhanyapanchaka & Amaatisara & Atisaradhikara-2/53 \\
\hline 5 & Panchamulyadi Yoga & Vataatisara & Atisaradhikara-2/56 \\
\hline 6 & Nagaradi Kashaya & Grahani & Grahanirogadhikara-3/49 \\
\hline 7 & Visvadya Churna & Grahani & Grahanirogadhikara-3/55 \\
\hline 8 & Pippalyadi Peya & Arsa & Arsodhikara-4/92 \\
9 & Bharngyadi Leha & Vataja Kasa & Kasadhikara-10/23 \\
\hline 10 & Satyadi Kwatha & Amavata & Amavatadhikara-22/16 \\
11 & Kwatha & Kukshi, Basti & Amavatadhikara-22/19 \\
\hline 12 & Panchakola Churna & Mandagni Shula & Amavatadhikara-27 \\
\hline 13 & Alambusadi Churna & Amavata & Amavatadhikara-22/29-33 \\
\hline 14 & Shunthi Ghirta & Vatarakta & Amavatadhikara-22/36-37 \\
\hline 15 & Chitrakadi Kwatha & Shula, Anaha, Gulma & Amavatadhikara-23/62 \\
\hline 16 & Haritakyadi Churna & Hridroga & Hrdrogadhikara-26/31 \\
\hline
\end{tabular}

\section{SHARANGDHAR SAMHITA: (13thCent.)}

Table no.10

\begin{tabular}{|l|l|c|c|} 
S.N & $\begin{array}{l}\text { Group } \\
\text { drugs/Preparations }\end{array}$ & $\begin{array}{c}\text { Indications/ } \\
\text { Action }\end{array}$ & References \\
\hline 1. & Shunthi Putapaka & $\begin{array}{c}\text { Amatisarajanya } \\
\text { Udarapida } \\
\text { Amajvara }\end{array}$ & Madhyamakhanda-1/39 \\
2. & Nagaradi Kwatha & Amatisara & Madhyamakhanda-2/9 \\
\hline 3. & Hriveradi Kwatha & Aruchi, Amadosa & Madhyamakhanda-2/68 \\
\hline 4. & Triyusana Churna & Amatisara & Madhyamakhanda-6/46 \\
\hline 5. & Shunthyadi Churna & $\begin{array}{c}\text { Pinasa, Swasa, Kasa, } \\
\text { Pratisyaya }\end{array}$ & Madhyamakhanda-7/22 \\
\hline 6. & Vyosadi Vati & Udararoga, Amavata & Madhyamakhanda-7/56 \\
\hline 7. & Mahayogaraja Guggulu & Grahani, Arsa & Madhyamakhanda-9/20 \\
\hline 8. & Changeri Ghrita & & \\
\hline
\end{tabular}


BHAVAPRAKASA: $\left(16^{\text {th }}\right.$ Century A.D $)$

Table no.11

\begin{tabular}{|l|l|c|c|} 
S.N. & Group of drugs/Preparations & Indications/Action & References \\
\hline 1. & Pachana Kashaya & Jvara & Chi.1/109 \\
\hline 2. & Satyadi Kwatha & Jvara & Chi.1/139 \\
\hline 3. & Vridhagangadhara Churna & Atisara & Chi.2/32 \\
\hline 4. & Hingvadi Churna & Kaphatisara & Chi.2/78 \\
\hline 5. & Chitrakadi Vati & Amapachana, Agnidipana, Grahani & Chi.4/52 \\
\hline 6. & Samasarkara Churna & Kaphaja Kasa & Chi.5/63 \\
\hline 7. & Pippalyadi Kwatha & Avabahuka & Chi.12/25 \\
\hline 8. & Masa taila & Amavata & Chi.24/84 \\
\hline 9. & Mahayogaraja Guggulu & Amavata & Chi.24/326 \\
\hline 10. & Hingavadya Churna & Amavata & Chi.26/31 \\
11. & Rasnasaptaka Kwatha & Amavata & Chi.6/43 \\
\hline 12. & Shunthi Ghrita & & Chi.26/80 \\
\hline
\end{tabular}

YOGARATNAKARA: $\left(17^{\text {th }}\right.$ Century A.D. $)$

Table no.12

\begin{tabular}{|l|l|c|c|}
\hline S.No. & $\begin{array}{l}\text { Group } \\
\text { drugs/Preparations }\end{array}$ & Indications/Action & References \\
\hline 1 & Guduchyadi Pachana & Vatajvara & Jvara Chikitsa \\
\hline 2 & Marichadi Churna & Vatajvara & Jvara Chikitsa \\
\hline 3 & Kwatha & Kaphajvara & Jvara Chikitsa \\
\hline 4 & Kwatha & Jvarapitta & Jvara Chikitsa \\
5 & Lavangadi Churna & Jvara, Gulma & Jvara Chikitsa \\
6 & Satpalaghrita & Manthara Jvara & Jvara Chikitsa \\
\hline 7 & Kwatha & Jvara & Jvara Chikitsa \\
\hline 8 & Churna & Ajirna & Jvara Chikitsa \\
\hline 9 & Sritakashaya & Amatisara & Atisara Chikitsa \\
\hline 10 & Srita & Jvaratisara & Atisara Chikitsa \\
\hline 11 & Laghugangadhara Churna & Vataja Grahani & Atisara Chikitsa \\
\hline 12 & Dadimavaleha & Pittaja Grahani & Grahani Chikitsa \\
\hline 13 & Shunthighrita & Sarva Grahani & Grahani Chikitsa \\
\hline 14 & Rasananadi Churna & Pittaja Grahani, Arsa & Grahani Chikitsa \\
\hline 15 & Shunthyadi Kwatha & Gulma, Udara Roga & Arsa Chikitsa \\
\hline 16 & Nagaradya Churna & Vasti, Kukshi Roga & Arsa Chikitsa \\
\hline 17 & Samasarkara Churna & Sarva Arsa & Arsa Chikitsa \\
\hline 18 & Shunthi Ghrita & Vatagulma, Shula & Ajirna Chikitsa \\
\hline 19 & Kankayana Gutika & Aittaja Kasa & Kajirna Chikitsa \\
\hline 20 & Bhaskaralavana Churna & & \\
\hline 21 & Sanjivani Gutika & Satyadi Kwatha & \\
\hline 22 & & & Kaitsa \\
\hline
\end{tabular}

BHAISAJYA RATNAVALI: $\left(18^{\text {th }}\right.$ Century A.D)

Table no.13

\begin{tabular}{|l|l|c|c|} 
S.No. & Preparations & Indications & References \\
\hline 1 & Sadanagapaniya & Vatika Jvara & Chi.5/26 \\
\hline 2 & Peya & Jvara & Chi.5/29 \\
\hline 3 & Mustaparpata kwatha & Jvara & Chi.5/68 \\
\hline 4 & Nagaradi Kwatha & Vatika Jvara & Chi.5/70 \\
\hline 5 & Kiratatiktadi Kwatha & Jvara & Chi.5/72 \\
\hline 6 & Kshudradi Kwatha & Vatika Jvara & Chi.5/73 \\
7 & Pippalimuladi Kwatha & Vatika Jvara & Chi.5/74 \\
\hline 8 & Bhunimbadi Kwatha & Vatika Jvara & Chi.5/85 \\
\hline 9 & Dasamuara & Chi.5/94 \\
\hline 10 & Visvadi Kwatha & Jvara & Chi.5/116 \\
\hline 11 & Nagaradi Kwatha & Paittika Jvara & Chi.5/124 \\
\hline
\end{tabular}




\begin{tabular}{|l|l|c|c|}
12 & Pachana Kwatha & Kaphaja Jvara & Chi.5/132 \\
\hline 13 & Nimbadi Kwatha & Kaphaja Jvara & Chi.5/136 \\
\hline 14 & Navanga Kwatha & Kaphaja Jvara & Chi.5/157 \\
\hline 15 & Ardrakadi Swaras & Sannipata Jvara & Chi.5/228 \\
\hline 16 & Katphaladi Kwatha & Sannipata Jvara & Chi.5/258 \\
\hline 17 & Yograja Kwatha & Abhinyasa Jvara & Chi.5/272 \\
\hline 18 & Karvyadi Kwatha & Vishama Jvara & Chi.5/344 \\
\hline 19 & Mahausadhadi Kwatha & Jvara & chi.5/350 \\
\hline 20 & Swalpa Bharangyadi Kwatha & Tridosaja, Jvara, Shula & chi.5/603 \\
\hline 21 & Saubhagya Vati & Taruna Jvara & chi.5/887 \\
\hline 22 & Jvara Murari Rasa & Amatisaraa, Jvara & Chi.6/5 \\
\hline 23 & Peya & Jvaratisara & Chi.6/8 \\
\hline 24 & Pathadi Kwatha & Jvara, Vamana, Aruchi & Chi.6/12 \\
\hline 25 & Churna & Pipasa-Atisara & Chi.7/10 \\
\hline 26 & Shunthyadi Kwatha & Shulayukta Atisara & Chi.7/16 \\
\hline 27 & Siddha Jala & Slesmatisara & Chi.7/46 \\
\hline 28 & Nagaradi Kwatha & Shotha, Jvara & Chi.7/103 \\
\hline 29 & Chavyadi Kwatha & Mandagni, Amakostha & Chi8/6 \\
\hline 30 & Narayana Churna & Amapachana & Chi8/11 \\
\hline 31 & Shunthyadi Kwatha & Arsagrahani, Pravahika & Chi8/29-31 \\
\hline 32 & Nagaradi Kwatha & Raktarsa & Chi9/32 \\
\hline 33 & Nagaradya Churna & Arsa & Chi9/33 \\
\hline 34 & Chandanadi Kwatha & Arsa & Chi9/37 \\
\hline 35 & Pathwadi Kwatha & Amavata & Chi29/16 \\
\hline 36 & Samasarkara Churna & Amavata & Chi29/22 \\
37 & Nagara Churna & Amavata & Chi29/23 \\
\hline 38 & Satyadi Kwatha & Janghoruparswatrika & Chi29/25 \\
39 & Rasonadi Kwatha & Prisithashul & \\
\hline 40 & Rasna Saptaka Kwatha & & Chi29/36 \\
\hline 41 & Shunthyadi Kwatha & & \\
\hline & & & \\
\hline
\end{tabular}

SHUNTHI DESCRIBED UNDER DIFFRENT
GANA/VARGA IN DIFFRENT NIGHANTUS:
\begin{tabular}{|l|c|} 
NAME OF NIGHANTU & VARGA \\
\hline Dhanvantri Nighantu & Shatapushpadi Varga \\
\hline Madhava Dravyaguna & Kanda Varga \\
\hline Madanpala Nighantu & Shunthyadi Varga \\
\hline Kaiyadeva Nighantu & Oshadhi Varga \\
\hline Bhavaprakash Nighantu & Haritakyadi Varga \\
\hline Raja Nighantu & Pippalyadi Varga \\
Priya Nighantu & Pippalyadi Varga \\
\hline Nighantu Adarsha & Adrakadi Varga \\
\hline
\end{tabular}

\section{NIRUKTI (DERIVATION OF NAME)}

Avakchatra - Leaves shaped like umbrella.

Ahicchatraka - Flower in radical spikes on long peduncleslike serpent's hood.

Katugranthi, Katubhadra, SringaveraRhizome is nodular, Tuberous and Pungent.

Utkata - Its smell is irritant.

Usana - It causes burning sensation.

Mahausadha, Visvabhesaja - It is universally reputed drug applicable in numerous disorders.

Visva - Assimilated quickly.

\section{SYNONYMS OF SHUNTHI IN VARIOUS TEXTS}

Amarakosa - Shunthi, Mahausadha, Visva, Nagara, Visvabhesaja.(2/9/38)

Medini Kosa - Visva, Shunthi

Sausruta Ni. - Sringavera, Kola, Nagara, Visvabhesaja, Shunthi, Katu, Usana (Pippalyadi150)

Astanga Ni -Shunthi, Mahausadha, Visva, Nagara, Visvabhesaja.

Paryaya Ratnamala - Visva, Mahausadha, Shunthi, Nagara, Visvabhesaja.

Madanadi Ni. - Shunthi, Mahausadha, Visvam, Nagara, Visvabhesaja, Visvausadh, Srngavera, Mahicchatra, Usana (Vellad4/5)

Dhanvantari Ni. - Shunthi, Mahausadha, Visva, Nagara, Visvabhesaja, Visvausadha, Srngavera, Katubhadra, Ardraka. (Satapuspadi Varga-2/82).

Sodhala Ni. - Shunthi, Mahausadha, Visva, Nagara, Visvabhesaja, Katubhanga, Vakcchatra, Srngavera, Ardraka (Satapuspadi Varga-340). 
Hrdaya Dipaka Ni. - Shunthi, Nagara, Mahausadha, Visvabhesaja, Visvam. (Dvipada Varga-69).

\section{Siddhamantra - Ardraka, Sringavera.}

(Kaphavataghna Varga-100).

Madanapala Ni. - Shunthi, Visvausadha, Katubhadra, Katutkata, Mahausadha, Sringavera, Nagara, Visvabhesaja. (Shunthyadirnama Varga2).

Kaiyadeva Ni. - Shunthi, Mahausadha, Visvausadha, Visvabhesaja, Nagara, Katubhadra, Rahucchatra, Katutkata (Ausadhi Varga-1150).

Bhava Prakasah Ni. - Shunthi, Visva, Visva, Nagara, Visvabhesaja, Usana, Katubhadram, Srngavera, Mahausadha (Haritakyadi Varga-44).

Raja Ni. - Shunthi, Mahausadha, Visva, Nagara, Visvabhesaja (Pippalyadi Varga- 24).

Shaligrama Ni. - Shunthi, Mahausadha, Visva, Suskadra, Visvabhesaja, Bhesaja, Srngavera, Visva, Nagara (Haritakyadi Varga- P.109).

\section{INTERPRETATION OF SYNONYMS}

Shunthi - Shunthatishoshayati kafamaamncheti shuthishoshne- having kafa and aa

Avakchatra - Leaves shaped like umbrella.

Ahicchatraka - Flower in radical spikes on long peduncleslike serpent's hood.
Utkata - Its smell is irritant.

Usana - It causes burning sensation

Katugranthi, Katubhadra, Sringavera-

Rhizome is nodular, Tuberous and Pungen

Mahausadha, Visvabhesaja - It is universally reputed drug applicable in numerous disorders.

Visva - Assimilated quickly.

\section{Synonnyms signifying morphology}

Avakchatra, Ahicchatraka, Katugranthi, Katubhadra, Sringavera.

\section{REGIONAL NAME OF SHUNTHI}

$\begin{array}{lll}\text { Hindi } & : & \text { Sontha, Ardraka, Adi } \\ \text { Beng. } & : & \text { Ada, Soonth } \\ \text { Mar. } & : & \text { Sunthi } \\ \text { Guj. } & : & \text { Sunth } \\ \text { Tel. } & : & \text { Sonthi, Ardrakamu } \\ \text { Tam. } & : & \text { Ksukku, Rukku } \\ \text { Urdu. } & : & \text { Adrak } \\ \text { Mar. } & : & \text { Ale } \\ \text { Bomb. } & : & \text { Adu } \\ \text { Pers. } & : & \text { Sangabir } \\ \text { Arab. } & : & \text { Zanjabil } \\ \text { English. } & : & \text { Dry ginger }\end{array}$

RASA, GUNA,VIRYA, VIPAKA AND KARMUKTA OF SUNTHI IN NIGHANTU GRANTHAS:

\begin{tabular}{|c|c|c|c|c|c|c|}
\hline Nighantu & Rasa & Guna & Virya & Vipaka & Varga & Prabhava/Karma \\
\hline Sausruta. Ni. & - & - & - & - & - & - \\
\hline Astanga Ni. & - & - & - & - & - & - \\
\hline Paryaya Ratnamala & - & - & - & - & - & - \\
\hline Madanadi Ni. & - & - & - & - & - & Grahi, Hridya, Dipana, Vrishya, Vibandhanut \\
\hline Dhanvantari Ni. & katu & Snigdha & Usna & Katu & $\begin{array}{l}\text { Satapuspadi } \\
\text { varga }\end{array}$ & $\begin{array}{c}\text { Vrishya, Sopha-Kapharuchihara, Vatodara, Swasa, } \\
\text { Pandu, Slipada Nasini }\end{array}$ \\
\hline Sodhala Ni. & - & - & - & - & - & - \\
\hline Hrdayadipaka Ni. & - & - & - & - & - & - \\
\hline Siddhamantra & - & - & - & - & - & - \\
\hline Madanapala Ni. & Katu & $\begin{array}{l}\text { Snigdh, } \\
\text { Laghu }\end{array}$ & Usna & Katu & Sunthyadi varga & Kaphavata, Amavata, Vibandhanut \\
\hline Kaiyadeva Ni. & Katu & $\begin{array}{c}\text { Snigdh, } \\
\text { Laghu }\end{array}$ & Usna & Madhura & Ausadhi Varga & Dipana, Vrsya, Sotha, Kaphavatajanya Roga \\
\hline Bhavaprakasa Ni & Katu & Snigdha & Usna & Madhura & $\begin{array}{l}\text { Haritakyadi } \\
\text { Varga }\end{array}$ & $\begin{array}{c}\text { Kaphavata, Vibandhanut, Vrsya, Swasa, Kasahara } \\
\text { etc. }\end{array}$ \\
\hline Raja Ni. & Katu & Snigdha & Usna & Katu & $\begin{array}{l}\text { Pippalyadi } \\
\text { Varga }\end{array}$ & Kaphasophanilapaha \\
\hline Saligrama Ni. & Katu & Snigdha & Usna & Madhura & $\begin{array}{l}\text { Haritakyadi } \\
\text { Varga }\end{array}$ & Shula, Swasa, Shlipada \\
\hline Priya Ni. & & & & & Pippayadi Varga & Vatanulomana Deepana, Pachana \\
\hline
\end{tabular}




\section{REFRENCES}

1. Charaka Samhita : Agnivesh, Revised by Charaka and Dridhabala,Vidyotini Hindi commentry by Pt. Kashi Nath Shastri and Gorakh Nath Chaturvedi, 13th Edition, 1986, Chaukhamba Sanskrit Series, Varanasi.

2. Sushruta Samhita : Hindi commentary by Kaviraj Ambika Dutt Shastri, 5th Edition, 1982, Chaukhamba Sanskrit Series, Varanasi. 